\title{
Erratum:
}

\section{Lie Groups, Lie Algebras, and Representations}

\author{
Brian C. Hall
}

(C) Springer International Publishing Switzerland 2015

B.C. Hall, Lie Groups, Lie Algebras, and Representations, Graduate Texts in Mathematics 222, DOI 10.1007/978-3-319-13467-3

DOI 10.1007/978-3-319-13467-3_14

The original version of this book was inadvertently published without the middle initial of the author's name as "Brian Hall". The correct name of the author should appear as "Brian C. Hall".

The online version of the original book can be found at http://dx.doi.org/10.1007/978-3-319-13467-3 